\title{
Obsessive-compulsive disorder and memory-mixing in temporal comparison: is implicit learning the missing link?
}

\author{
Bon-Mi Gu ${ }^{*}$ and Keshav Kukreja ${ }^{2}$ \\ Department of Psychology and Neuroscience, Duke University, Durham, NC, USA \\ 2 Department of Neuroscience, Rhodes College, Memphis, TN, USA \\ *Correspondence: bg43@duke.edu
}

Humans cope with uncertainty in their daily lives in a variety of ways. At one end of the spectrum would be individuals diagnosed with obsessive-compulsive disorder (OCD). OCD patients have difficulty tolerating even the slightest amount of uncertainty if it is related to their symptoms (see Gentes and Ruscio, 2011 for review). For example, a patient may wash his hands continuously for an hour to avoid germs, check the door repeatedly to ensure that it is locked safely, or hoard everything for the slight chance that they might need it later. In contrast, healthy individuals are able to ignore these uncertainties because they exploit their prior experiences, providing themselves with a sense of security. Acknowledging the reasons for their anxiety as trivial does not necessarily attenuate obsessive thoughts, and deficits in implicit processes dealing with uncertainty could be a potential reason for their sustained obsession. Dysfunctions in implicit learning have been reported in patients with OCD (Deckersbach et al., 2002; Kathmann et al., 2005), suggesting a possible role of implicit contextual knowledge in reducing the anxiety underlying obsessive behaviors.

The application of implicit knowledge could be useful in terms of optimizing behavioral performance in many cases, but it can also bias our behavior in unfavorable ways. For example, if a seasoned squash player were learning to play tennis for the first time, he would perform better than a novice due to the level of similarity between these two sports with regard to fine hand and wrist coordination. However, because he is accustomed to the shorter squash racquet, it would be difficult for him to adjust to the noticeably longer tennis racquet. Therefore, although this implicit knowledge would most likely increase the player's precision in performance by reducing the variability of hand movements, it would sacrifice accuracy due to the inher- ent differences between the racquet sizes and the required motor actions of grip balance and strength.

It was recently demonstrated that a Bayesian model, which optimizes reasoning through the trade-off between accuracy and precision, could simulate the biased performance of participants in situations where they are instructed to reproduce specific stimulus durations (Jazayeri and Shadlen, 2010; Mamassian and Landy, 2010). Participants tend to bias their reproductions toward the mean of the distribution; specifically, they overestimate "short" durations and underestimate "long" durations. This phenomenon was first referred to as Vierordt's law (Woodrow, 1951; Lejeune and Wearden, 2009; Mamassian and Landy, 2010) and, more recently, has been described as a form of "memory-mixing," which represents the distortions in temporal memory caused by the encoding of multiple signal durations into a single memory distribution (Penney et al., 1998, 2000; Klapproth, 2009; Grondin, 2010; Gu and Meck, 2011).

The reasons for the existence of "memory-mixing" remain largely unknown, although instructional ambiguity has been proposed as a possible explanation (Klapproth, 2009) and factors dealing with the scalar property of temporal memory may also be involved (Gibbon et al., 1984). Another plausible explanation is that the implicit knowledge of the underlying stimulus distribution generates behaviors that are less variable, but still biased. Devoting less attention to the encoding of the immediate stimulus could result in the reliance on prior context through automatic processing, thus generating a form of "memorymixing." If "memory-mixing" is in fact generated by implicit knowledge of prior contexts and reliance on automatic processing, we could then hypothesize that patients with OCD, who are known to have deficits in implicit learning (Deckersbach et al., 2002; Kathmann et al., 2005), will display a lesser degree of "memory-mixing" on a temporal reproduction/comparison task in comparison to healthy participants.

Furthermore, there is evidence showing that patients with OCD could compensate for their deficiency in implicit learning with hippocampal-dependent explicit knowledge (Rauch et al., 2007). A combination of normal explicit learning and a deficit in implicit learning would suggest that an OCD patient's perception of the current trial's signal duration would be less influenced by the context of prior signal durations. Therefore, it is highly probable that they compensate for their inability to exploit implicit knowledge of the context (e.g., trial sequence and mixture of different signal durations) with increased vigilance to the present signal duration that they are asked to encode. This will eventually reduce the degree of uncertainty as well as the effect of "memory-mixing."

Nevertheless, we cannot discount the possibility that the "memory-mixing" could be caused by other factors. Instead of the implicit knowledge of the entire distribution of prior signal durations, the residual of the immediately preceding duration could be the main source of "memory-mixing." The effects of the most recent trial have been shown to significantly influence the subsequent trial (Gu and Meck, 2011), implicating a process whereby temporally close events have a greater effect on the current trial. If this is the case, then the strength of the residual components of the previous trial will determine the resulting degree of "memory-mixing."

Cognitive inflexibility in OCD patients has been observed through deficits in task-switching and reversal learning (Chamberlain et al., 2008; Gu et al., 2008). These effects imply that the mental rigidity of OCD patients causes them to maintain their previous memory sets, which creates difficulty in rapidly updating memory with new information. Therefore, patients 
with OCD will display stronger residuals from previous trials during their performance on the subsequent trial, resulting in an increase of "memory-mixing." Furthermore, it is expected that the attention of OCD patients would be especially captured by information encoded during the previous trial if it is related to feedback and/or has an emotional valence (see Droit-Volet and Meck, 2007; Gu et al., 2011). For example, if a neutral and OC-related emotional stimulus are presented in combination with 1.0 and $0.6 \mathrm{~s}$ durations, respectively, it is likely that the reproduction of the durations associated with the neutral stimulus preceded by the shorter emotional stimulus would be underestimated.

In conclusion: We have hypothesized two scenarios whereby "memory-mixing" would be expected to occur within a temporal reproduction/comparison task for patients with OCD who are thought to have altered dopaminergic activity in the cortico-striatal circuits involved in timing and time perception (Buhusi and Meck, 2005; Meck et al., 2008; Allman and Meck, 2011; Gu et al., 2011). It is possible that OCD patients would exhibit more "memory-mixing" than control participants due to the strong residuals from the previous trial. However, it would be more plausible that they exhibit a lesser degree of "memory-mixing" due to their inability to exploit implicit knowledge while simultaneously displaying increased vigilance toward the signal presented on the current trial. This would be especially likely if a sufficiently long inter-trial interval is presented without the intervention of any emotional stimuli. The potential significance of this hypothesis lies within its ability to aid in our understanding of how normal individuals and OCD patients deal with uncertainty in their environment. We necessarily choose a balance between utilizing our accumulated implicit knowledge and increasing our vigilance to systematic changes in the environment (Buhusi and Meck, 2009; Gu et al., 2011).

\section{REFERENCES}

Allman, M. J., and Meck, W.H. (2011). Pathophysiological distortions in time perception and timed performance. Brain (in press).

Buhusi, C. V., and Meck, W. H. (2005). What makes us tick? Functional and neural mechanisms of interval timing. Nat. Rev. Neurosci. 6, 755-765.

Buhusi, C.V., and Meck, W.H. (2009). Relative time sharing: new findings and an extension of the resource allocation model of temporal processing. Philos. Trans. R. Soc. Lond. B. Biol. Sci. 364, 1875-1885.

Chamberlain, S. R., Menzies, L., Hampshire, A., Suckling, J., Fineberg, N. A., del Campo, N., Aitken, M., Craig, K., Owen, A. M., Bullmore, E. T., Robbins, T. W., and Sahakian, B. J. (2008). Orbitofrontal dysfunction in patients with obsessive-compulsive disorder and their unaffected relatives. Science 321 , 421-422.

Deckersbach, T., Savage, C. R., Curran, T., Bohne, A., Wilhelm, S., Baer, L., Jenike, M. A., and Rauch, S. L. (2002). A study of parallel implicit and explicit information processing in patients with obsessive-compulsive disorder. Am. J. Psychiatry 159, 1780-1782.

Droit-Volet, S., and Meck, W. H. (2007). How emotions colour our perception of time. Trends Cogn. Sci. (Regul. Ed.) 11, 504-513.

Gentes, E. L., and Ruscio, A. M. (2011). A meta-analysis of the relation of intolerance of uncertainty to symptoms of generalized anxiety disorder, major depressive disorder, and obsessive-compulsive disorder. Clin. Psychol. Rev. 31, 923-933.

Gibbon, J., Church, R. M., and Meck, W.H. (1984). Scalar timing in memory. Ann. N. Y. Acad. Sci. 423, 52-77.

Grondin, S. (2010). Timing and time perception: a review of recent behavioral and neuroscience findings and theoretical directions. Atten. Percept.Psychophys 72,561-582.

Gu, B. M., and Meck, W.H. (2011). "New perspectives on Vierordt's law: memory-mixing in ordinal temporal comparison tasks," in Time and Time Perception 2010 LNAI 6789, eds A. Vatakis, A. Esposito, F. Cummins, G. Papadelis, and M. Giagkou (Berlin: Springer-Verlag), 67-78.

Gu, B. M., Park, J. Y., Kang, D. H., Lee, S. J., Yoo, S. Y., Jo, H. J., Choi, C. H., Lee, J. M., and Kwon, J. S. (2008). Neural correlates of cognitive inflexibility during task-switching in obsessive-compulsive disorder. Brain 131, 155-164.
Gu, B. M., Cheng, R. K., Yin, B., and Meck, W. H. (2011). Quinpirole-induced sensitization to noisy/sparse periodic input: temporal synchronization as a component of obsessive-compulsive disorder. Neuroscience 179, 143-150.

Jazayeri, M., and Shadlen, M. N. (2010). Temporal context calibrates interval timing. Nat. Neurosci. 13, 1020-1026.

Kathmann, N., Rupertseder, C., Hauke, W., and Zaudig, M. (2005). Implicit sequence learning in obsessive-compulsive disorder: further support for the fronto-striatal dysfunction model. Biol. Psychiatry 58, 239-244.

Klapproth, F. (2009). Single-modality memory mixing in temporal generalization: an effect due to instructional ambiguity. Neuroquantology 7, 85-94.

Lejeune, H., and Wearden, J. H. (2009). Vierordt's "the experimental study of the time sense" (1868) and its legacy. Eur. J. Cogn. Psychol. 21, 941-960.

Mamassian, P., and Landy, M. S. (2010). It's that time again. Nat. Neurosci. 13, 914-916.

Meck, W. H., Penney, T. B., and Pouthas, V. (2008). Cortico-striatal representation of time in animals and humans. Curr. Opin. Neurobiol. 18, 145-152.

Penney, T. B., Allan, L. G., Meck, W. H., and Gibbon, J. (1998). "Memory mixing in duration bisection," in Timing of Behavior: Neural, Psychological and Computational Perspectives, eds D. A. Rosenbaum and C. E. Collyer (Cambridge, MA: MIT Press), 165-193.

Penney, T. B., Gibbon, J., and Meck, W. H. (2000). Differential effects of auditory and visual signals on clock speed and temporal memory. J. Exp. Psychol. Hum. Percept. Perform. 26, 1770-1787.

Rauch, S. L., Wedig, M. M., Wright, C. I., Martis, B., McMullin, K. G., Shin, L. M., Cannistraro, P. A., and Wilhelm, S. (2007). Functional magnetic resonance imaging study of regional brain activation during implicit sequence learning in obsessive-compulsive disorder. Biol. Psychiatry 61, 330-336.

Woodrow, H. (1951). “Time perception,” in Handbook of Experimental Psychology, ed. S. S. Stevens (New York, NY: Wiley), 1224-1236.

Received: 26 July 2011; accepted: 30 July 2011; published online: 18 August 2011.

Citation: Gu B-M and Kukreja K (2011) Obsessivecompulsive disorder and memory-mixing in temporal comparison: is implicit learning the missing link? Front. Integr. Neurosci. 5:38. doi: 10.3389/fnint.2011.00038 Copyright ( $(2011$ Gu and Kukreja. This is an open-access article subject to a non-exclusive license between the authors and Frontiers Media SA, which permits use, distribution and reproduction in other forums, provided the original authors and source are credited and other Frontiers conditions are complied with. 